\title{
Light scattering by complex ice-analogue crystals
}

\author{
Zbigniew Ulanowski ${ }^{\mathrm{a}^{*}}$, Evelyn Hesse ${ }^{\mathrm{a}}$, Paul H. Kaye ${ }^{\mathrm{a}}$ and Anthony J. Baran ${ }^{\mathrm{b}}$ \\ ${ }^{a}$ Science and Technology Research Institute, University of Hertfordshire, Hatfield, Herts AL10 9AB, UK \\ ${ }^{b}$ Met Office, Cordouan 2, Fitzroy Road, Exeter EX1 3PB, UK
}

\begin{abstract}
Angle-dependent light scattering measurements on single ice analogues crystals are described. Phase functions and degree of linear polarization are measured for electrodynamically levitated crystals. A procedure for randomizing particle orientation during levitation is demonstrated. The dependence of scattering on the shape, complexity and surface roughness of the crystals is examined. The phase functions from complex crystals with smooth surfaces show little dependence on shape. There is close agreement between the measured functions and the analytic phase function for ice clouds. However, rosettes with rough surfaces have qualitatively different phase functions, with raised side and back scattering. The asymmetry parameter is typically about $0.8 \pm 0.04$ and $0.63 \pm 0.05$ for smooth and rough crystals, respectively. The $22^{\circ}$ halo peak is present for smooth rosettes and aggregates but absent for rough rosettes. Two-dimensional scattering patterns from several crystals in fixed orientations are also shown. The results suggest that it may be possible to use such patterns to discriminate not only between crystals of different shape but also to obtain some information on surface properties.
\end{abstract}

Keywords: Light scattering; Ice crystals; Analogues; Levitation; Cirrus; Asymmetry parameter; Roughness

\section{Introduction}

The significance of ice and mixed-phase clouds to the earth-atmosphere radiation balance and climate is well established. However, present understanding of cirrus concerning scattering properties of ice crystals is weak, which is mainly due to inadequate theoretical models and lack of knowledge concerning in situ size and shape. The areas of applicability of current models are uncertain with respect to the size parameter, crystal shape and surface roughness. For larger size parameters and/or complex shapes accurate models do not exist, have not yet been adequately verified or are computationally very demanding. Therefore, measurements of the scattering properties of ice crystals are of paramount importance if the magnitude and sign of feedback on climate change due to radiative forcing by cirrus is to be quantified. Field measurements of scattering on ice in clouds are not only difficult but for correct interpretation they require the knowledge of the size and shape of the crystals being observed. This information is imprecise, especially for smaller sizes. Laboratory measurements on ice crystals are also very demanding, as the particles have to be maintained in equilibrium with water vapour at low temperatures. Moreover, control over ice crystal morphology is poor[1,2]. The measurement of scattering on nonspherical particles poses further challenges, e.g. the need to present the particle in a specific orientation or, more appropriately, a set of representative orientations. Hence, the particle should be immobilized on the one hand, preferably using a non-contact method, and its alignment should be adjustable on the other hand. Here, a useful technique is electrodynamic levitation, where gravity is counteracted by forces produced by a set of electrodes[1,2,3].

We recently circumvented the difficulties associated with measurements on real ice crystals by the use of materials that are stable at room temperature, have the correct refractive index at visible wavelengths $(\approx 1.31)$, crystallize in similar habits to ice[4,5] and produce geometrically-correct halos[6]. We now extend these measurements to scattering from crystals with random orientation, which is more relevant to atmospheric ice. The randomization is achieved by taking advantage of angular oscillations in an electrodynamic balance (EDB) levitation trap [3,5]. In order to constrain estimates of the asymmetry parameter, which depends strongly on forward scattering, the lower scattering angle limit has been reduced from $3^{\circ}$ to about $0.5^{\circ}$. We present results of angle-dependent 
light scattering measurements on levitated ice-like crystals, including phase functions and degree of linear polarization, and two-dimensional scattering patterns from crystals supported by thin fibres or glass plates. In this study we focus on the dependence of scattering on the shape and surface roughness of the crystals.

\section{Methods}

Light scattering measurements were carried out using a fibre-optics laser diffractometer (LD) covering the range of scattering angles from 3 to $177^{\circ}$. A multi-line Ar-ion laser was the light source and a prism was used to select the wavelength. A dual-wavelength half-wave plate was used to obtain two states of polarization, perpendicular and parallel to the horizontal scattering plane[7]. Low scattering angle measurements were achieved using a linear photodiode array with 512 pixels $25 \mu \mathrm{m}$ by $250 \mu \mathrm{m}$ in size (Reticon RL0512K). A small mirror placed within the laser beam acted as a baffle protecting the array from direct illumination - see Fig. 1. Typical scattering angle range was approximately $0.5^{\circ}$ to $5^{\circ}$. The array was placed on the opposite side of the laser beam from the fibreoptics detector, so that, strictly speaking, the scattering angle range was $359.5^{\circ}$ to $355^{\circ}$. This arrangement is acceptable if the forward scattering is symmetric, as can be expected for randomly oriented particles.

The ice analogue crystals were grown in solution. Typically, a few $\mathrm{ml}$ of saturated solution of sodium fluorosilicate was poured into a $50 \mathrm{~mm}$ diameter glass Petri dish and allowed to evaporate to dryness at in a laminar flow cabinet at room temperature. Individual crystals were selected using a fine needle or a specially constructed microgripper attached to a micromanipulator. In order to obtain rough surfaces a precipitate of sodium fluorosilicate containing fine, submicrometer particles was dripped onto single crystals held with the microgripper and allowed to dry; the procedure was repeated up to three times. While the procedure for producing the analogues is relatively simple, caution is required because sodium fluorosilicate is classified as toxic, and can cause severe irritation of eyes and mucous membranes. Also, it must be noted that impurities present in the solution, including undissolved fluorosilicate, can affect crystal habit.

The ice-analogue crystals were levitated using a specially developed electrodynamic balance. Micromanipulation techniques were used to select single crystals of desired shape and size, and to inject them into the EDB. In addition a method for crystal recovery after measurements was developed, so that further examination became possible, e.g. using scanning electron microscopy[3]. Images of particles during levitation were obtained using CCD cameras and microscope objectives with a numerical aperture of 0.16 in the horizontal, and 0.4 in the vertical (along trap axis) direction, respectively. Orientational randomization was achieved by taking advantage of angular oscillations in the EDB. Angular instability of particles in EDBs was investigated and modelled by Hesse et al.[3]. When such instability is produced, non-spherical objects first undergo angular oscillations and eventually, when entering deeper into instability conditions, rotate or show apparently chaotic angular motions[5]. For some combinations of particle properties and trapping conditions, most notably low trapping field strength and frequency, axial motion instability may occur first. In this case linear oscillations dominate over angular ones.

Two-dimensional scattering patterns were recorded using a $612 \mathrm{~nm}$ wavelength $\mathrm{He}-\mathrm{Ne}$ laser, a translucent projection screen and a digital CCD camera. A column-shaped ice-analogue crystal was glued to a $6 \mu \mathrm{m}$ diameter carbon fibre which was in turn glued to a stainless steel tube attached axially to a rotation stage. Complex crystals were placed on antireflection-coated windows.

\section{Phase functions and asymmetry parameter}

Fig. 2 shows an ice analogue aggregate crystal with a maximum dimension of $93 \mu \mathrm{m}$ and Fig. 3 the same crystal during levitation. The randomized orientation images in Fig. 3 (c) and (d) demonstrate that the randomization was satisfactory, especially around the vertical axis: the elliptical shape of the averaged image (d) is probably caused by residual linear oscillation in the image plane. Partial alignment is apparent in Fig. 3 (c). While it should be possible to reduce the extent of this residual alignment by making the instability stronger, this carries the danger of particle loss as the motions 
become increasingly chaotic and eventually axial instability sets in. Furthermore, such residual alignment would be unlikely to lead to phase function distortion for particles with complex shapes. On the other hand, account must be taken of the highly non-uniform spatial distribution of scattered intensity from faceted objects, which is dominated by specular reflections and equally narrow refracted "beams". This is illustrated in Fig. 4 where scattered intensity measured for a smooth rosette in the EDB trap is shown for 50 different orientations. The non-uniformity demands that a sufficient number of measurements is taken to ensure good averaging.

Fig. 5 shows a normalized phase function for the $93 \mu \mathrm{m}$ aggregate, averaged over 500 orientations. Accurate normalization of phase functions and the estimation of the asymmetry parameter $g$ require the knowledge of the full angular range of scattering. The present measurements extend down to a scattering angle of about $0.5^{\circ}$. Since scattering at such low angles is dominated by diffraction, for randomly oriented particles it is largely independent of shape and Lorenz-Mie theory can be used to extrapolate the scattered intensity, assuming an ensemble of spheres with average cross-section area equal to that of the aggregate in randomized orientation. We have used this procedure to obtain $g$ for the aggregate in Figs. 2-3 and found a value of $0.81 \pm 0.03$. However, for highly nonspherical particles the use of Lorenz-Mie theory may not be appropriate and should ideally be replaced with an approach that takes into account the (changing) shape of the particle as it rotates during the averaging process. Since exact approaches are computationally very demanding for large size parameters, a compromise is to use Fraunhofer diffraction on an ensemble of equal average cross-section area, randomly oriented elliptical apertures, which can be solved analytically[8]. In the small angle approximation the diffracted intensity for a randomly oriented ellipse can be written as a function of scattering angle $\theta$

$$
\Psi(\theta)=\left(\frac{2 \pi a b}{k}\right)^{2} \int_{0}^{2 \pi}\left(\frac{J_{1}(k \beta)}{\beta}\right)^{2} d \phi
$$

where $a$ and $b$ are the semiaxes of the ellipse, $J_{1}$ is the Bessel function of the first kind, $k$ is the wavenumber and

$$
\beta=\sqrt{(a \theta \cos \phi)^{2}+(b \theta \sin \phi)^{2}} .
$$

The integration is carried out over the azimuthal angle $\phi$ to randomize the orientation of the ellipse. This approach has been used to extrapolate the measured scattering function for some of the crystals studied here, including a five-branched, $93 \mu \mathrm{m}$ maximum dimension rosette shown in Fig. 6: its phase function is displayed in Fig. 7. The normalized phase functions for six complex crystals in random orientation, including smooth aggregate and rosettes and rough rosettes are shown in Fig. 8, together with values of the asymmetry parameter calculated from the phase functions. Optical microscopy images of the rough rosettes are shown in Fig. 9. The degree of linear polarization, averaged separately for the smooth and rough crystals, is given in Fig. 10. The ice-analogue aggregate phase function shown in Fig. 5 is compared in Fig. 11 to the analytic phase function for ice clouds, which is a modification of the Henyey-Greenstein phase function[9]. The choice of parameters of the analytic function is based solely on the knowledge of $g$.

\section{Two-dimensional scattering patterns}

Two-dimensional scattering patterns obtained from a hexagonal column fixed to a thin fibre are shown in Fig. 12 for ten different orientations. Patterns obtained from the same column are examined in more detail in a companion paper (this issue), in comparison with computations, together with computed patterns for several other crystal shapes[10]. Two-dimensional scattering patterns for fixed orientation of the $93 \mu \mathrm{m}$ aggregate and smooth and rough rosettes shown in Fig. 13 are displayed in Fig. 14.

\section{Discussion and conclusions}

For the first time, phase functions from levitated particles with randomized orientation have been obtained. Measurements down to the scattering angle of $0.5^{\circ}$ make it possible to accurately normalize the phase functions and to calculate the asymmetry parameter. The narrow forward scattering range 
can be extrapolated using Mie theory or Fraunhofer diffraction on an ensemble of equal average crosssection area ellipses. The present measurements on complex analogue crystals, together with previous results[4], show phase functions which are flat at larger scattering angles, in common with other types of non-spherical particles. The phase functions from complex crystals with smooth surfaces show remarkably little dependence on shape; the asymmetry parameter $g$ is consistently at about $0.8 \pm 0.04$. The $22^{\circ}$ halo peak is present for both rosettes and aggregates. There is close agreement between the measured functions and the analytic phase function for ice clouds[9]. However, the rosettes with rough surfaces have qualitatively different phase functions, characterized by increased side and back scattering, and much lower asymmetry parameter - typically about $0.63 \pm 0.05$ - than the smooth crystals. The difference between the two values of $g$ is statistically significant at the $90 \%$ confidence level. A further difference is that no halo peak is present for the rough crystals (Fig. 7). Absence of halos has also been noted for computed phase functions for such objects[11]. The absence of the halo makes crystals with rough surfaces a possible candidate for cirrus ice. The unexpectedly low value of the asymmetry parameter would then have far-reaching consequences for the magnitude of cirrus cloud radiative forcing. This area clearly deserves further study.

Two-dimensional scattering patterns recorded for different crystal habits show attributes that can be related to the geometry of the crystals. The smooth rosette pattern stands out most, with clear arcs that can be attributed, by analogy with the patterns for the single column, to individual arms of the rosette. At first sight the patterns produced by the smooth aggregate and the rough rosette appear similar. However, close examination reveals that there are significant differences. Most notably the aggregate pattern contains several bright spots at scattering angles below $45^{\circ}$, while the rough rosette pattern is azimuthally symmetric in this region (Fig. 14). These distinguishing features may provide information on surface roughness of crystals. This indicates that two-dimensional scattering could be used as a basis for discrimination between important classes of ice crystals. Additional means of discrimination could be provided by measurements of polarization: for example, smooth and rough crystals exhibit clear differences in the 20 to $35^{\circ}$ region (Fig. 10).

Finally, while the propensity of smooth crystals to produce scattering patterns that are very nonuniform spatially may aid their discrimination, it can also lead to difficulties with cloud particle counters due to the possibility of multiple triggering of detection optics, and hence overcounting. The nature of the two-dimensional patterns shown here suggests that such overcounting might be more prevalent for instruments that detect scattering at larger angles and/or over narrow apertures. Instruments in the latter category would also be subject to large sizing errors.

\section{Acknowledgements}

This work was supported by the Natural Environment Research Council. Anthony Baran was partly funded by the UK Department of the Environment Food and Rural Affairs under contract PECD $7 / 12 / 37$.

\section{References}

[1] Pluchino A. Observations of halo scattering from single ice crystals. Opt Lett 1986;11:276-278.

[2] Bacon NJ, Swanson BD. Laboratory measurements of light scattering by single levitated ice crystals. J Atm Sci 2000;57:2094-2104.

[3] Hesse E, Ulanowski Z, Kaye PH. Stability characteristics of cylindrical fibres in an electrodynamic balance designed for single particle investigation. J Aerosol Sci 2002;33:149-163.

[4] Ulanowski Z, Hesse E, Kaye PH, Baran AJ, Chandrasekhar R. Scattering of light from atmospheric ice analogues. J Quantit Spectr Rad Transf 2003;79-80C:1091-1102.

[5] Ulanowski Z, Hesse E, Kaye PH. Ice Scattering Experiments. http://strc.herts.ac.uk/ls/ise.html University of Hertfordshire, Hatfield, 2005.

[6] Ulanowski Z. Ice analog halos. Appl Opt 2005;44:5754-5758.

[7] Ulanowski Z, Greenaway RS, Kaye PH, Ludlow IK. Laser diffractometer for single particle scattering measurements. Meas Sci Technol 2002;13:292-296. 
[8] Kathuria Y. Far-field radiation patterns of elliptical apertures and its annuli. IEEE Trans Antennas Propag 1983;31:360-364.

[9] Baran AJ, Francis PN, Labonnote L-C, Doutriaux-Boucher M. A scattering phase function for ice cloud. Q J Royal Met Soc 2001;127:2395-2416.

[10] Clarke AJM, Hesse E, Ulanowski Z, Kaye PH. 3D implementation of ray tracing combined with diffraction on facets: Comparisons to SVM and experiment with a demonstration of its particle characterization capability. J Quantit Spectr Rad Transf 2006 (in press, this issue).

[11] Yang P, Liou KN. Single-scattering properties of complex ice crystals in terrestrial atmosphere. Contr Atmos Phy 1998;71:223-248. 


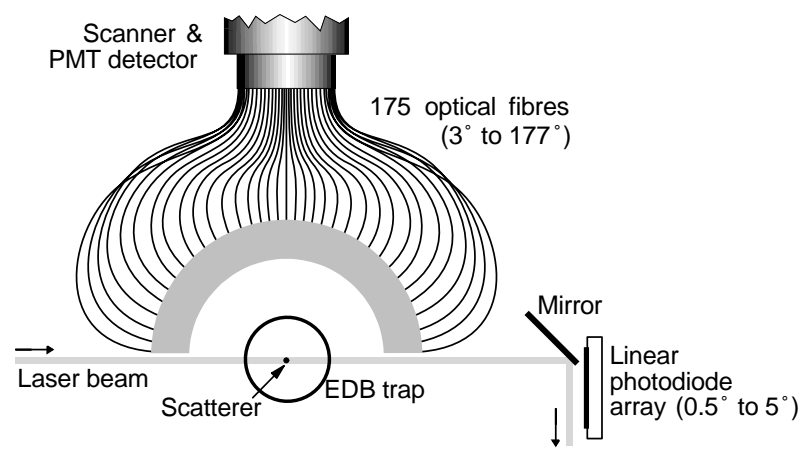

Fig. 1. Experimental setup for measuring light scattering from levitated crystals (view from above).

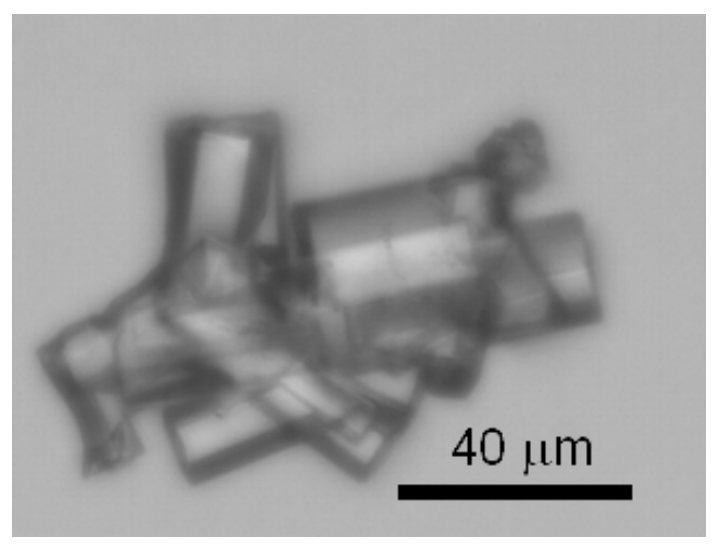

Fig. 2. Optical microscopy image of an ice-analogue aggregate with a maximum dimension of $93 \mu \mathrm{m}$.

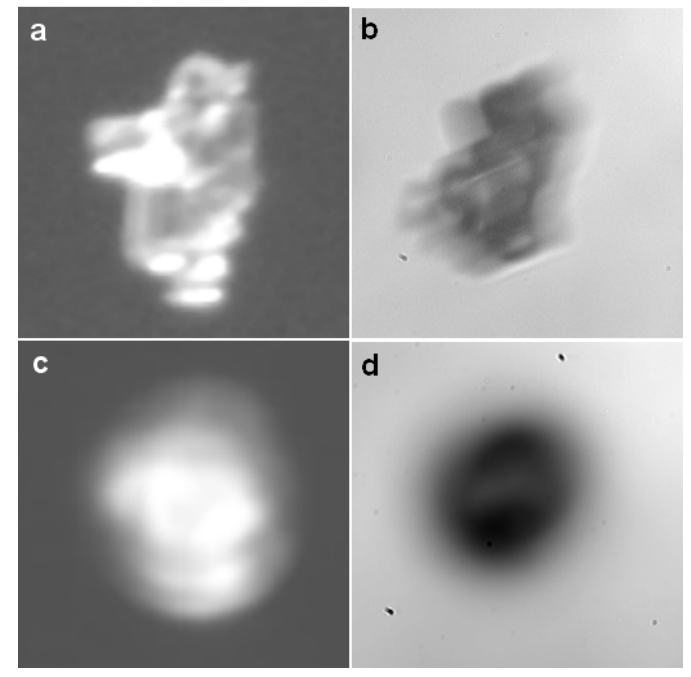

Fig. 3. Images of the aggregate crystal from Fig. 2 during levitation. The upper images are for a nearly motionless crystal, the lower ones were obtained by averaging over about 30 seconds during angular randomization. The left-hand image pair is from a horizontally-looking camera with dark-field illumination, and the right-hand pair from a vertically-looking bright-field camera. 


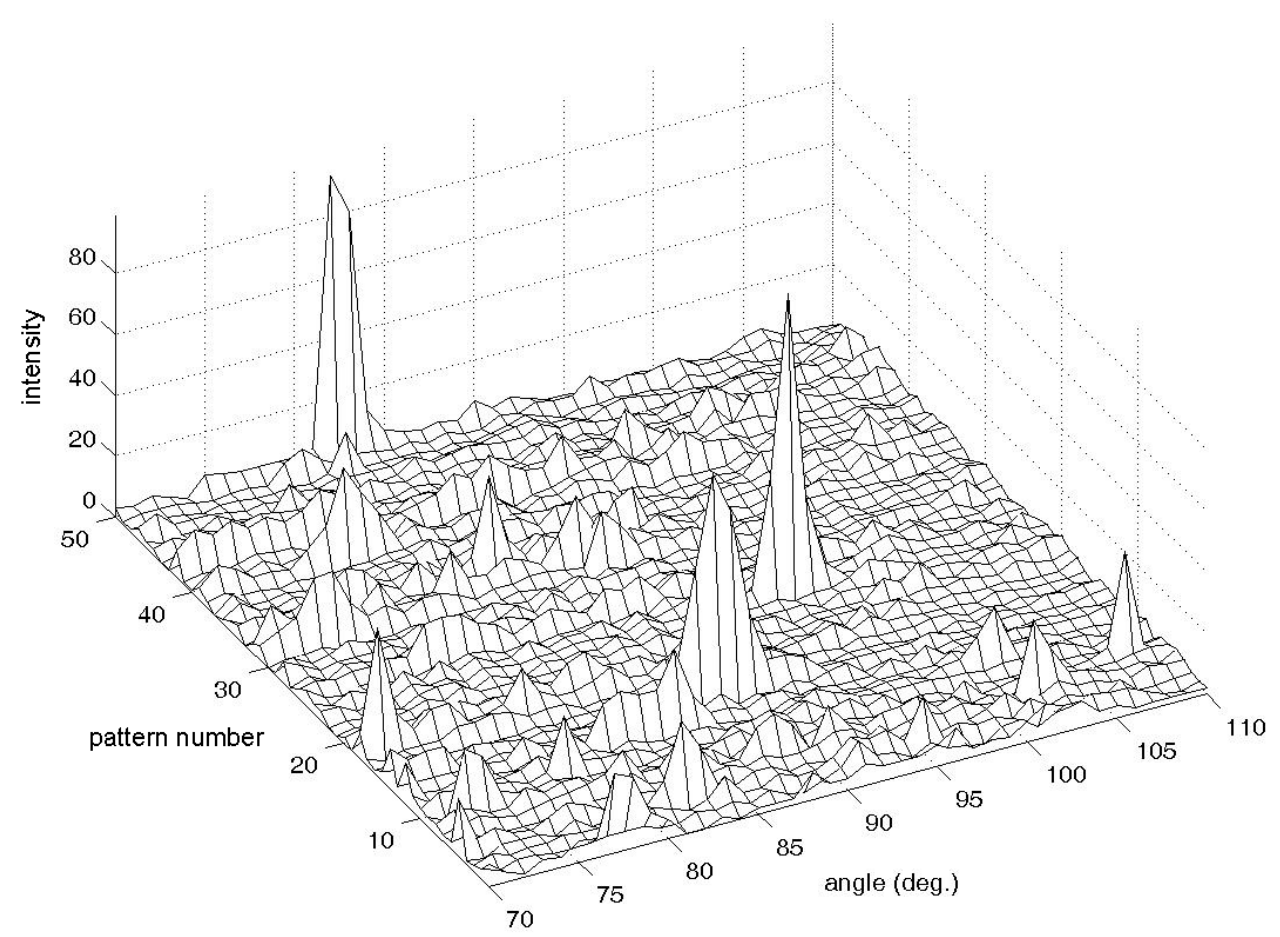

Fig. 4. Scattered intensity for a smooth 8-branch rosette with a maximum dimension of $72 \mu \mathrm{m}$, measured in one plane for 50 different orientations in the EDB trap. The scattering angle range is 70 to $110^{\circ}$.

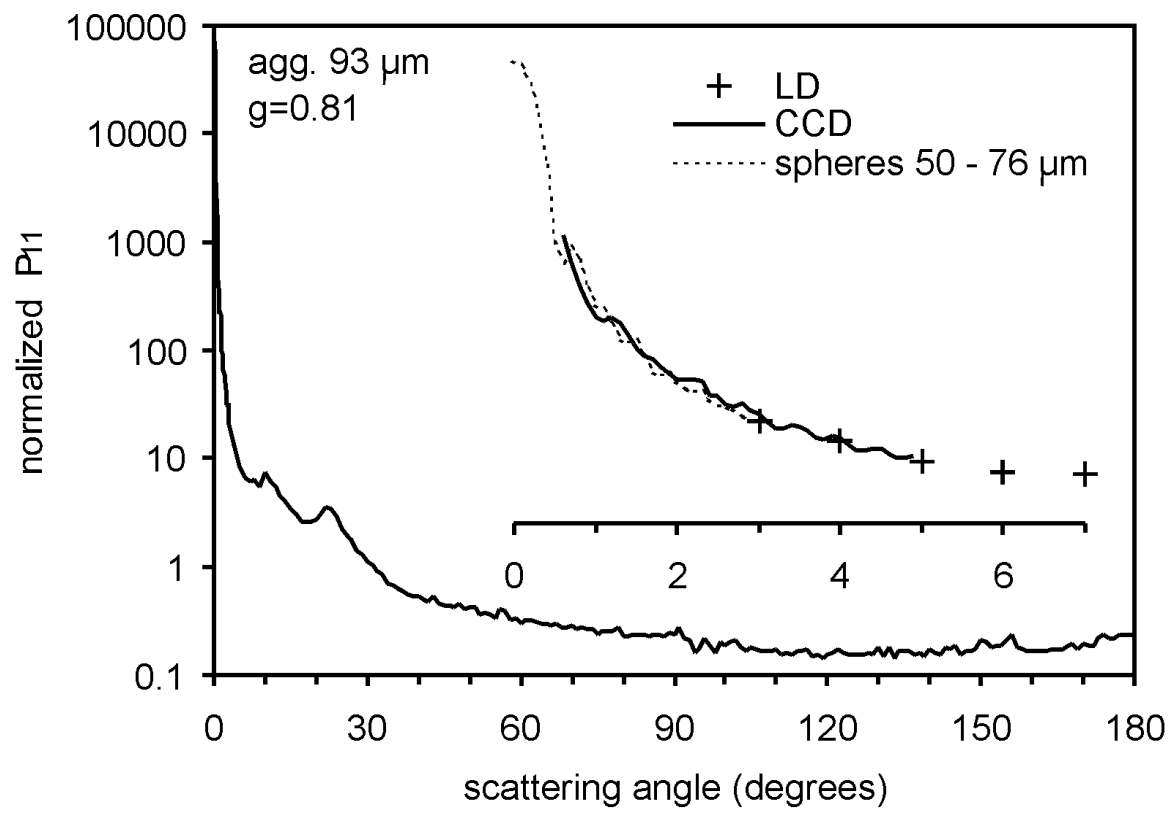

Fig. 5. Normalized phase function for the $93 \mu \mathrm{m}$ ice-analogue aggregate at $514.5 \mathrm{~nm}$ wavelength. The results were extrapolated between 0 and $0.5^{\circ}$ using Lorenz-Mie theory for an ensemble of equal average cross-section area ice spheres (diameters uniformly distributed between 50 and $76 \mu \mathrm{m}$ ). The inset, with the same vertical scale as the main graph, shows for angles $0-7^{\circ}$ the relationships between the laser diffractometer results (LD), the diode array results $(\mathrm{CCD})$ and the theory-extrapolated data. 


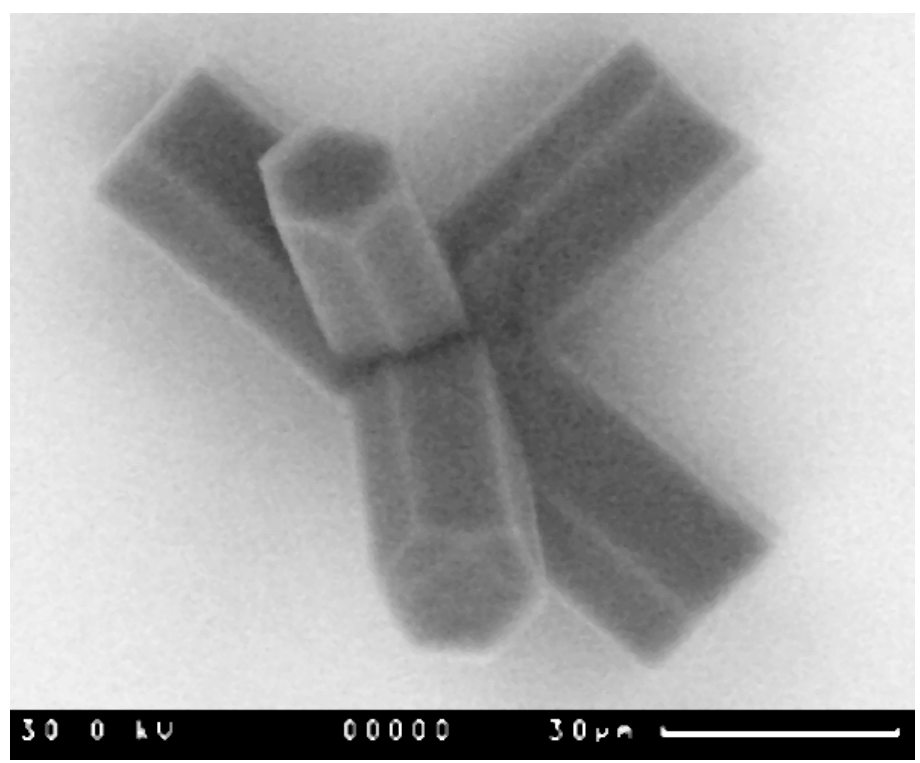

Fig. 6. Scanning electron microscopy image of $93 \mu \mathrm{m}$ ice-analogue rosette recovered from the EDB trap following measurements. The sample is not coated.

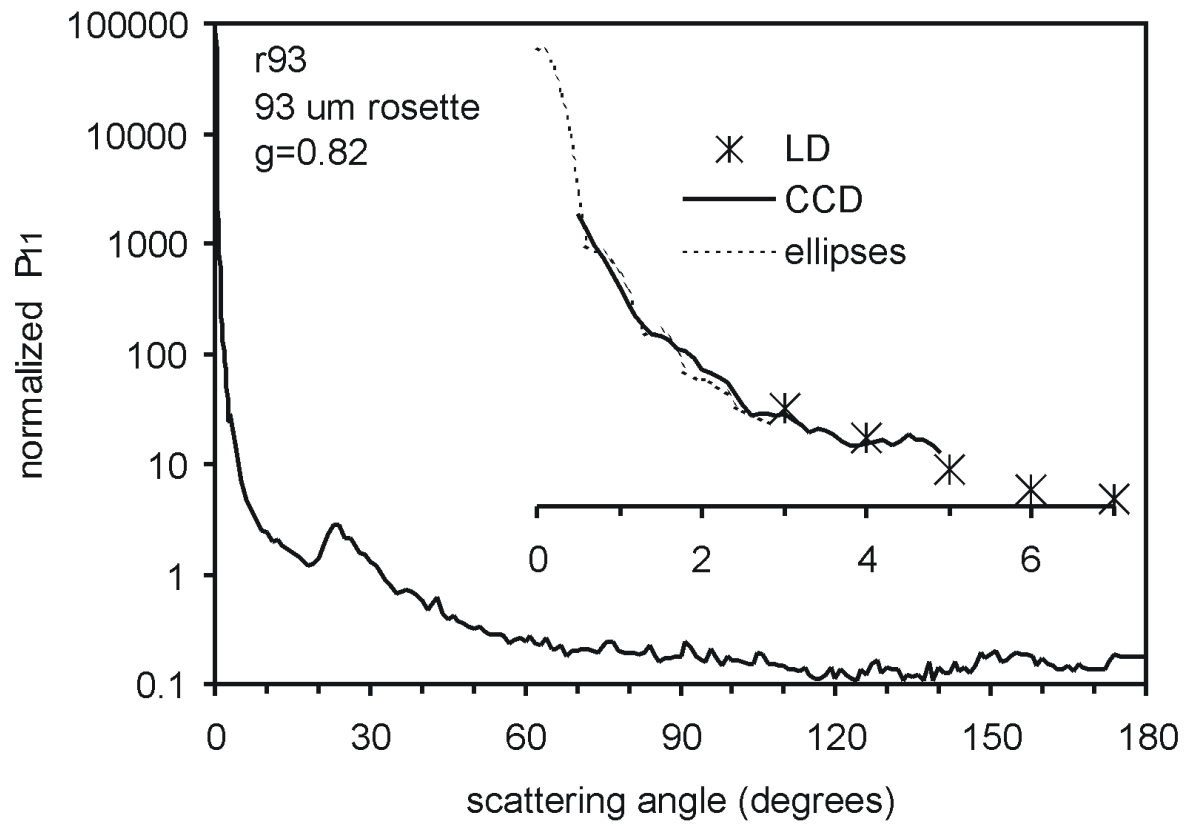

Fig. 7. Normalized phase function for the $93 \mu \mathrm{m}$ ice-analogue rosette from Fig. 6, measured at $514.5 \mathrm{~nm}$ wavelength. The results were extrapolated between 0 and $0.5^{\circ}$ using Fraunhofer diffraction on an ensemble of equal average cross-section area ellipses. The inset, with the same vertical scale as the main graph, shows for angles $0-7^{\circ}$ the relationships between the laser diffractometer results (LD), the diode array results $(\mathrm{CCD})$ and the theory-extrapolated data. 


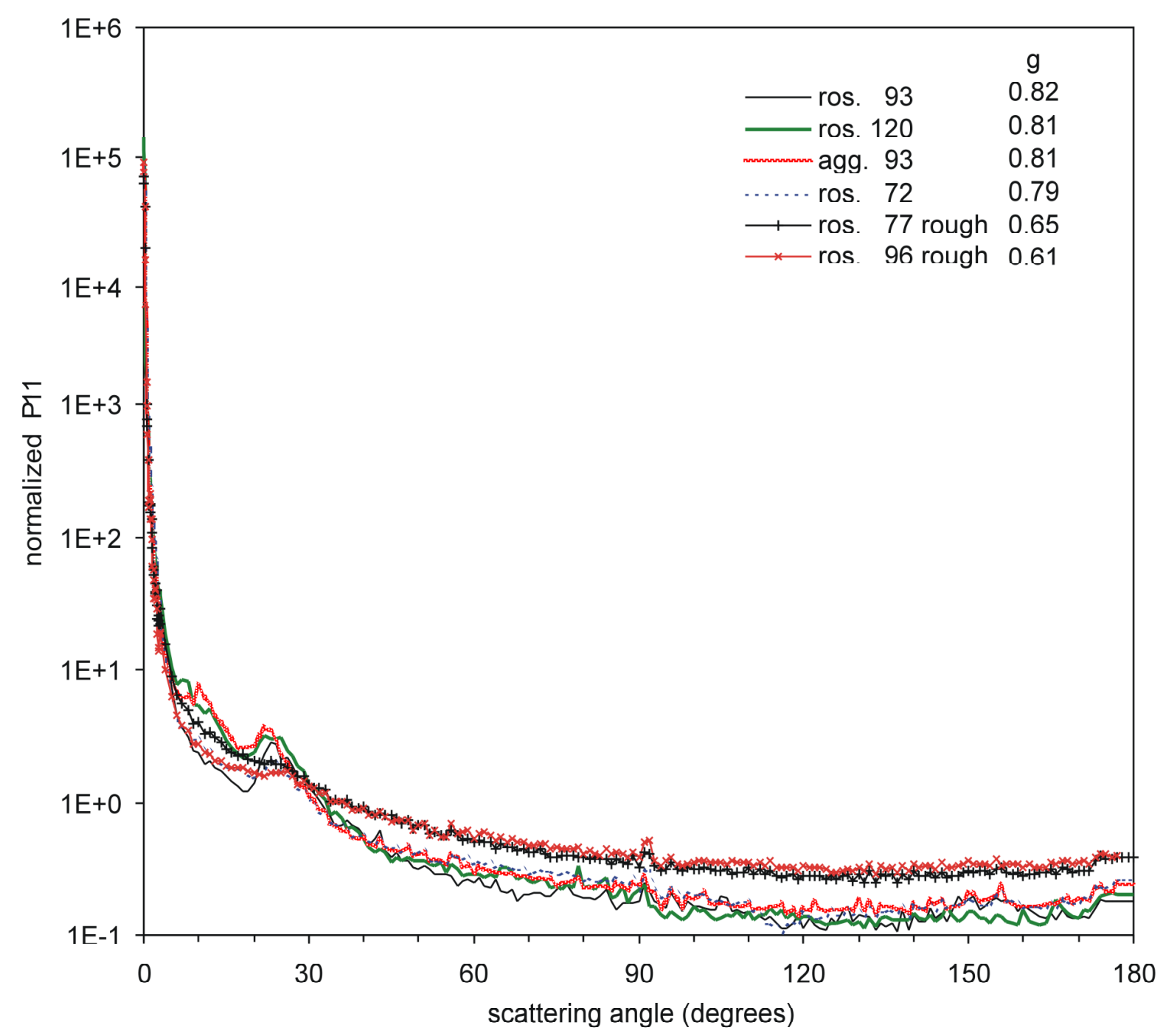

Fig. 8. Normalized phase functions for the ice-analogue aggregate and five rosettes, including two rough ones, at $514.5 \mathrm{~nm}$ wavelength, in random orientation. The maximum dimension of each crystal in micrometers and the value of the asymmetry parameter $g$ are shown in the legend.

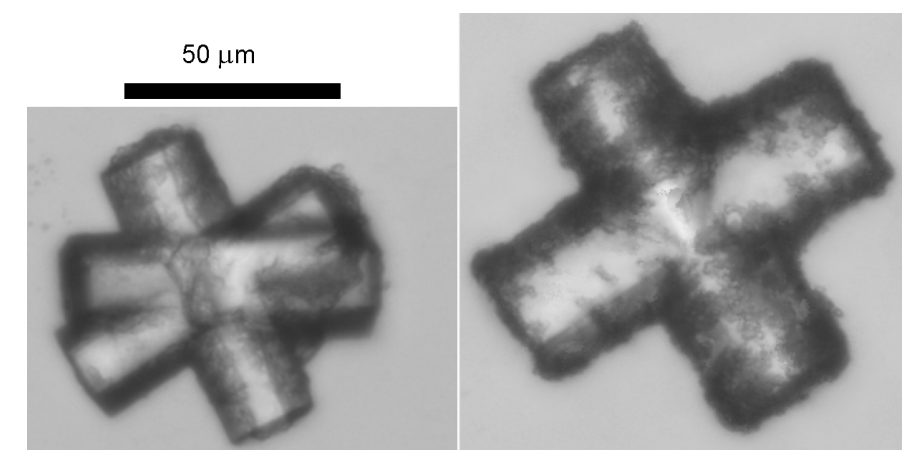

Fig. 9. Optical microscopy image of ice-analogue rosettes with rough surfaces and maximum dimension of $77 \mu \mathrm{m}$ (left) and $96 \mu \mathrm{m}$ (right). 


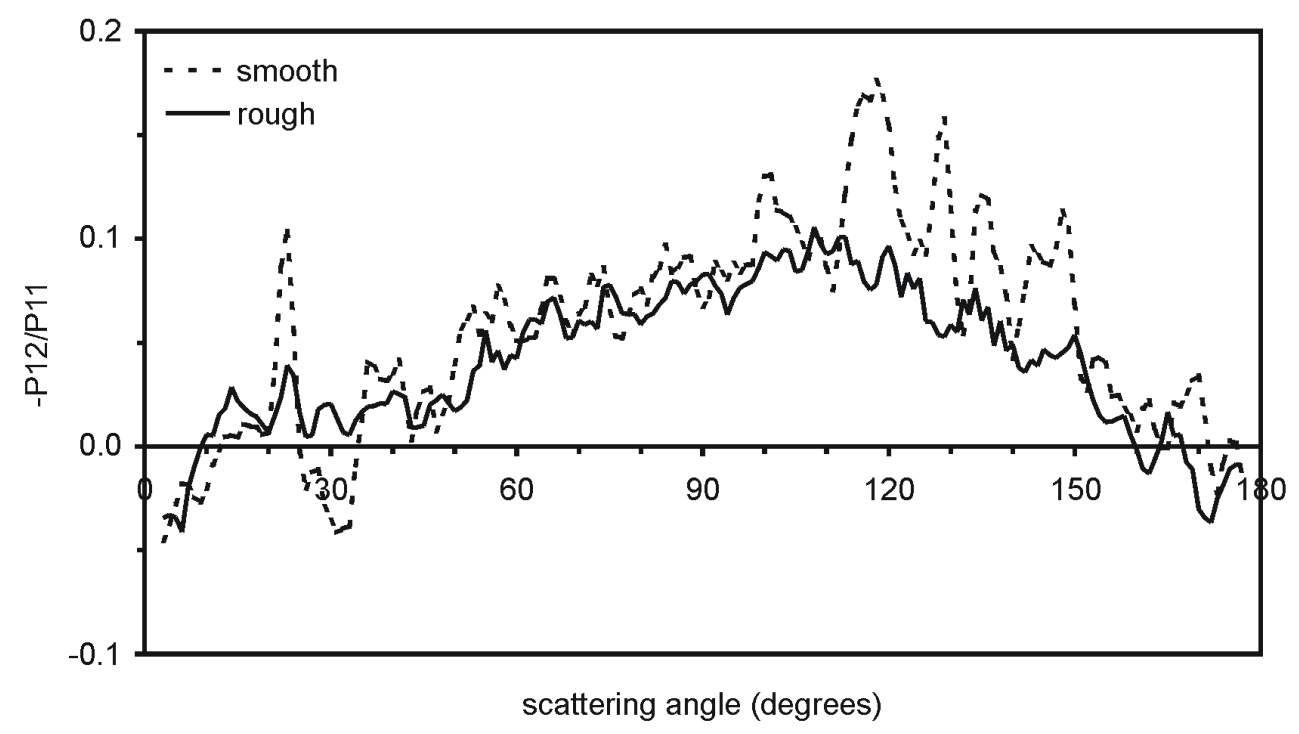

Fig. 10. Average degree of linear polarization for four smooth and two rough ice-analogue crystals identified in Fig. 8, in random orientation.

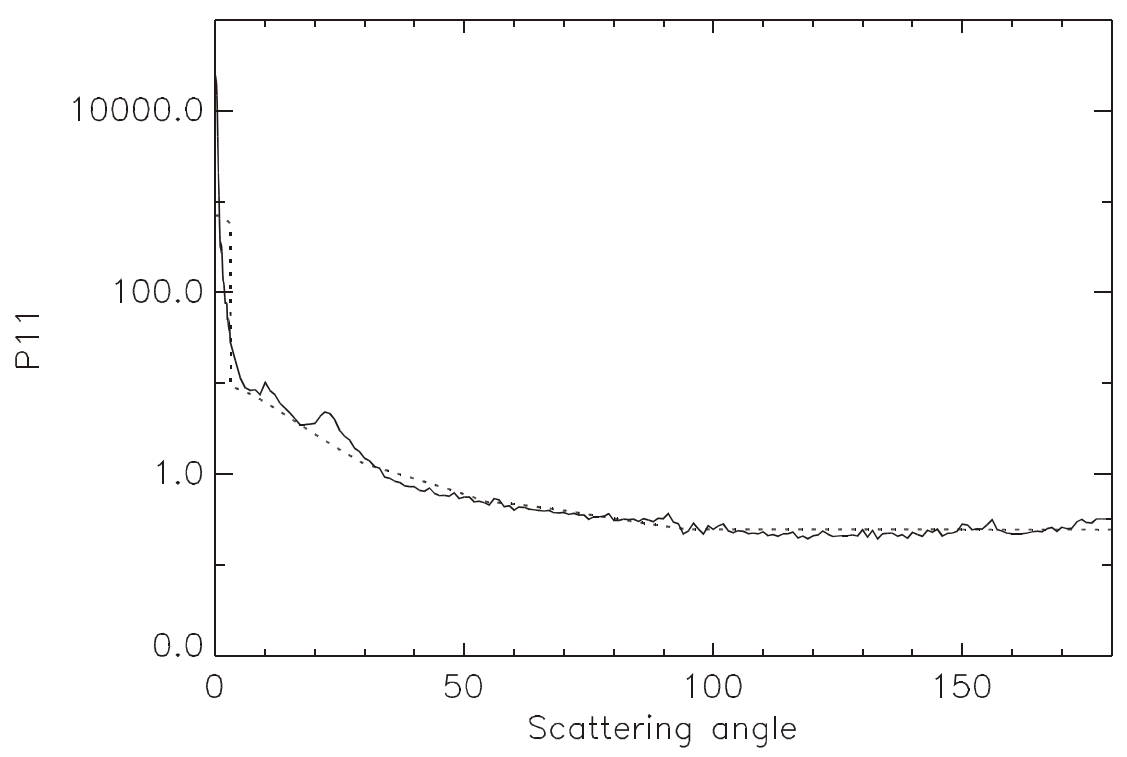

Fig. 11. Normalized phase function for the $93 \mu \mathrm{m}$ aggregate in random orientation (solid line) compared to the analytic phase function due to Baran et al.[9] (dotted line). 


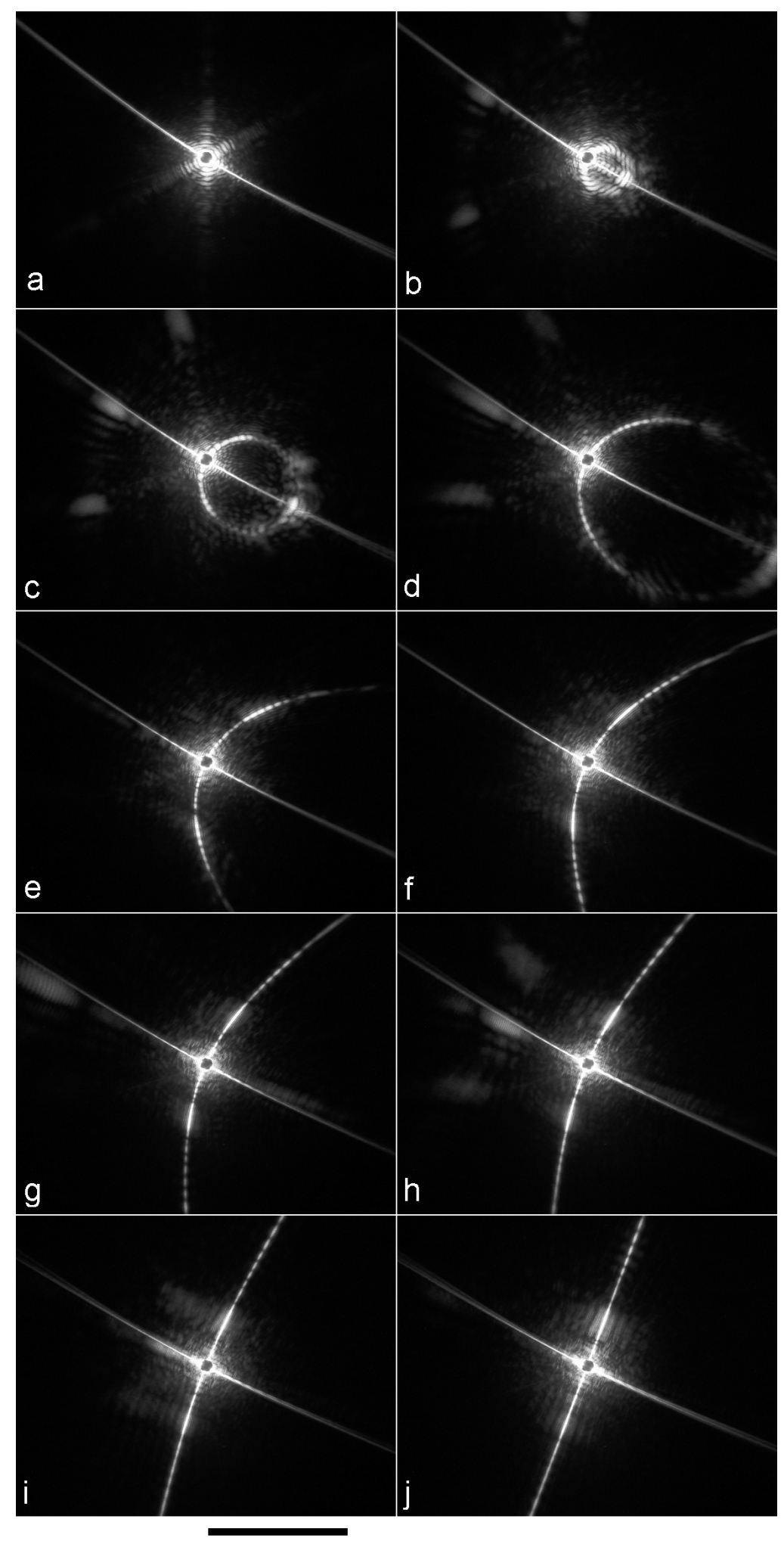

Fig. 12. Two-dimensional scattering patterns obtained from a hexagonal ice-analogue column $61 \mu \mathrm{m}$ long and $15 \mu \mathrm{m}$ in diameter mounted on a thin carbon fibre. In the image sequence the column was rotated in $10^{\circ}$ increments from parallel (image a) to perpendicular (image j) orientation with respect to the incident beam direction. The scattering from the fibre apppears as a diagonal line running from the upper left to the lower right corner of each image. This line is approximately perpendicular to the axis of rotation. The centre of each pattern corresponds to forward scattering and the distance from the centre to the tangent of the scattering angle $\theta$, the scale bar at the bottom represents $\tan \theta=1$. 


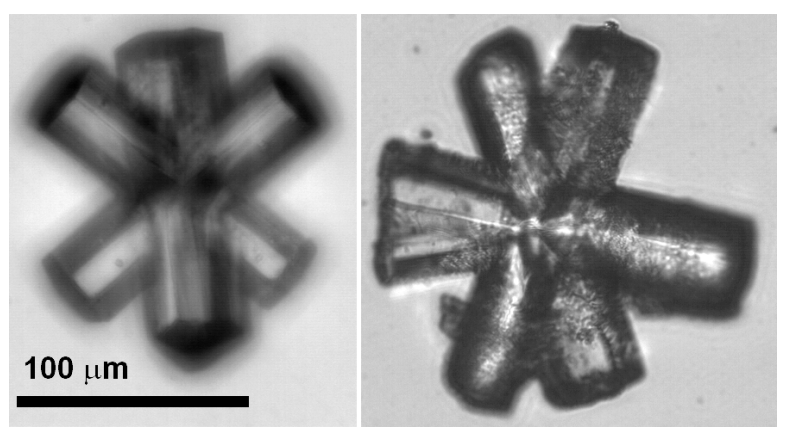

Fig. 13. Optical microscopy images of smooth (left) and rough (right) ice analogue rosettes.
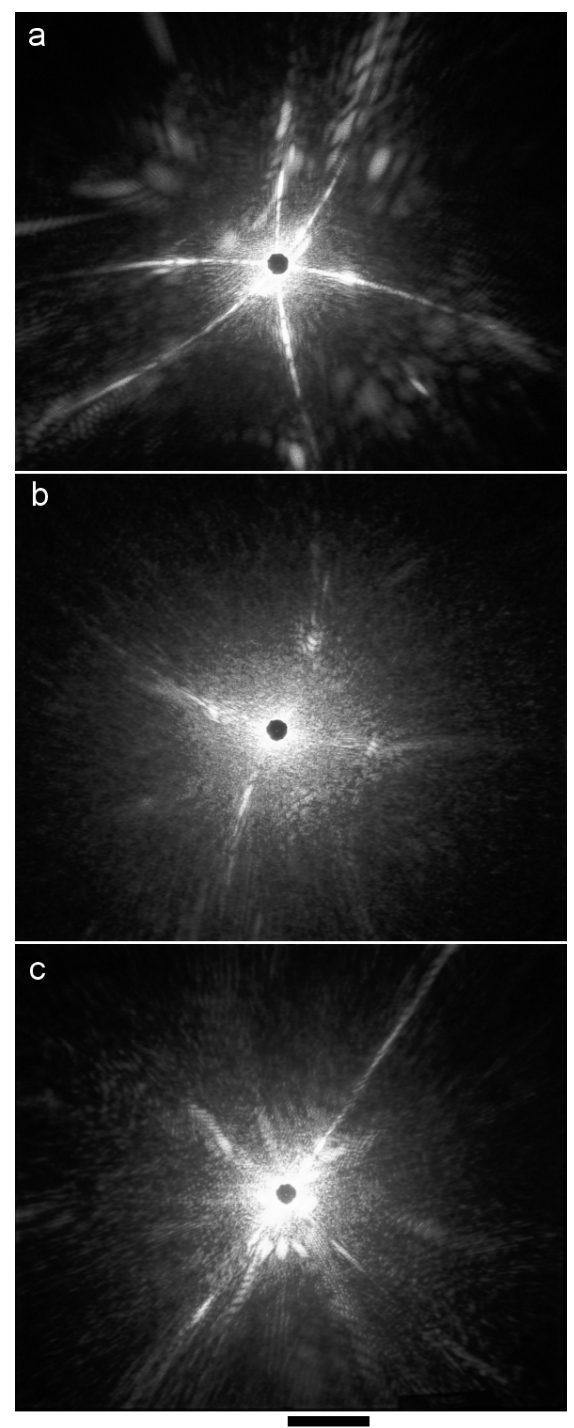

Fig. 14. Two-dimensional scattering patterns from: (a) a smooth $145 \mu \mathrm{m} 6$ arm rosette (showing clear contributions from the individual branches), (b) $160 \mu \mathrm{m}$ rough rosette of similar shape, both shown in Fig. 13, and (c) the 93 $\mu \mathrm{m}$ aggregate from Figs. 2-3, all on anti-reflection coated glass plates. The centre of each pattern corresponds to forward scattering and the distance from the centre to the tangent of the scattering angle $\theta$, the scale bar at the bottom represents $\tan \theta=1$. 